\title{
Assessment of Facial Homogeneity with Regard to Genealogical Aspects Based on Deep Learning Approach
}

\author{
Ravi Kumar Y B $\mathbf{B}^{1}$, Narayanappa $\mathbf{C ~ K}^{2}$, Dayananda $\mathbf{P}^{3}$ \\ ${ }^{1}$ Research Scholar, Department of Computer Science \& Engineering, RIT, Affiliated to VTU, Bangalore \\ ${ }^{2}$ Associate Professor, Department of Medical Electronics Engineering, RIT, Affiliated to VTU, Bangalore \\ ${ }^{3}$ Professor, Department of Information Sciences \& Engineering, JSS Academy of Technical Education, Bangalore, \\ India
}

Article History: Received: 10 November 2020; Revised: 12 January 2021; Accepted: 27 January 2021; Published online: 05 April 2021

\begin{abstract}
The current research work encompasses the assessment of similarity based facial features of images with erected method so as to determines the genealogical similarity. It is based on the principle of grouping the closer features, as compared to those which are away from the predefined threshold for a better ascertainment of the extracted features. The system developed is trained using deep learning-oriented architecture incorporating these closer features for a binary classification of the subjects considered into genealogic non-genealogic. The genealogic set of data is further used to calculate the percentage of similarity with erected methods. The present work considered XX datasets from XXXX source for the assessment of facial similarities. The results portrayed an accuracy of $96.3 \%$ for genealogic data, the salient among them being those of father-daughter $(98.1 \%)$, father-son(98.3\%), mother-daughter(96.6\%), mother-son(96.1\%) genealogy in case of the datasets from "kinface W-I". Extending this work onto "kinface W-II" set of data, the results were promising with father-daughter (98.5\%), father-son(96.7\%), mother-daughter(93.4\%) and mother-son(98.9\%) genealogy. Such an approach could be further extended to larger database so as to assess the genealogical similarity with the aid of machine-learning algorithms

Keywords: Computer Science, Features extraction, Features learning Clustering, Facial similarities, Classification
\end{abstract}

\section{Introduction}

Machine learning based approach is known to be successful in the assessment of facial similarities, verification and associated applications. Such features are often useful in the assessment of genealogical aspects with regard to the quantity of facial homogeneity in the subjects considered. The features extracted from the facial images are often subjected to novel assessment approaches so as to obtain the desired classification of the datasets considered. This approach has been extensively adopted in various image processing as well as in case of psychophysical applications with EEG [4]. The present work has been successful in the assessment of the genealogical aspects with regard to the facial homogeneity ascertained from the facial features. Similar features are brought closer to each other and the rest are pushed away from each other in the sample space. Squeeze net modules are used to train the system with images of size $127 \times 127$ being the basic structure. The resized images are then used for the learning module and feature extraction, which improves the accuracy of the system.

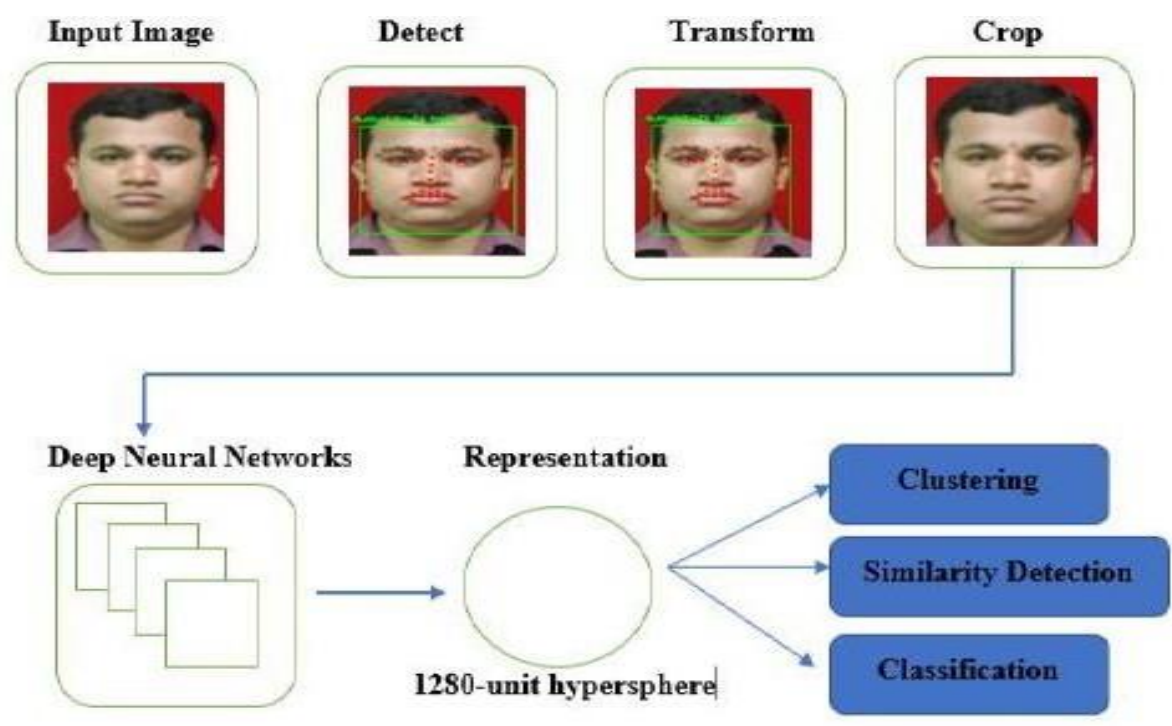

Figure 1. Overall architecture of the erected method with different phases of processing of input image 


\section{Background}

The discriminative subspaces of color components of Laiadi, Oualid et.al of [1] have been useful in extracting the facial features of images by analysing the color components in sub-spaces. However, the accuracy has been an issue due to the usage of image subspaces for the information calculations. Deep compact similarity metrics of Xiuzhuang Zhou et.al [2] has been more efficient due to the incorporation of the same. The drawbacks in this case have been mitigated with the erected method approach. Miguel Bordallo Lopez et.al of [3] determined the facial kinships by incorporating the hierarchical features representation learning. In further literatures pertaining to facial recognition, various approaches have been developed so as to ascertain the neural abilities of recognition. The present research work highlights the facial feature assessment for the genealogical identification.

\section{Deep Learning Features For Facial Similarities Verification}

The facial features of source image are compared with all other facial features of dataset by incorporating the designated Deep Learning facial features to make the system more expanded to combine the Existing Squeeze Net features. The features of facial images are cropped into 127x127 sizes before feeding the system with input images. The output of squeeze net is concatenated with Deep Learning Features Extraction (DLFE) that makes the system more efficient and yields good results.

The features extraction by Deep Learning concatenated with Sqeeze Net features learning makes the system more efficient and robust by computing the information of facial features gathered from eq. (2), where eq. (1) is a generalized representation of the system.

$$
S(v, h ; \theta)=V_{\mathrm{i}}-B_{\mathrm{i}}-H_{\mathrm{i}}
$$

Weights $W_{\mathrm{ij}}$ of the proposed Deep Learning Features Extraction (DLFE) does the task of calculating the information of facial features of one photograph with target images buy incorporating the formulation of eq (1) consisting of source image represented by $v_{\mathrm{i}}$ with respect to the target image indicated by $h_{\mathrm{j}}$.

$$
S(v, h ; \theta)=\sum_{\mathrm{i}=1 \mathrm{j}=1}^{N} \sum_{\mathrm{i}}^{M} \mathrm{~W}_{\mathrm{ij}} h_{\mathrm{j}}-\sum_{\mathrm{i}=1}^{N} b_{\mathrm{i}} v_{\mathrm{i}}-\sum_{\mathrm{j}=1}^{M} a_{\mathrm{j}} h_{\mathrm{j}}
$$

Eq.(3) measures the sequential information obtained from generalized Squeeze Net features with Deep Learning Features Extraction (DLFE) thereby the system increases the measure of features of one source image with target image.

$$
S(v, h ; \theta)=-V^{T} \mathrm{~W} h-b^{T} V-a^{T} h
$$

This concatenated information is expanded into more statistical features learning that makes the system more suitable for measuring the information gathered from features of images to be compared with target images that makes the system more efficient.

$$
S(v, h ; \theta)=-\sum_{\mathrm{i}}^{N} \sum_{\mathrm{j}}^{M} \bar{\sigma}_{\mathrm{i}} \mathrm{W}_{\mathrm{ij}} h_{\mathrm{j}}-\sum_{\mathrm{i}=1}^{N} \frac{\left(v_{\mathrm{i}}-b_{\mathrm{i}}\right)_{2}}{2 \sigma^{2}}-\sum_{\mathrm{j}=1}^{M} \underset{\mathrm{j}}{a} \mathrm{j}_{\mathrm{j}}
$$

Statistical system of eq. (4) plays a significant role in measuring the features by deep neural information that makes the system and concatenates with measured weights to make the system more efficient and robust. The system has helped the proposed method with weights with exponential information obtained with weighted features learning as per eq. (5)

$$
\begin{aligned}
& S(v, h)=\frac{1}{Z} \exp (-S(v, h ; \theta)) \\
& S(v)=\sum_{h} S(v, h)
\end{aligned}
$$

Eq. (6) of the proposed method makes the system more efficient by concatenating with Squeeze Net features obtained from processing of images as per fig. 1 


\section{Algorithm (DLFE)}

Deep Learning Features Extraction (DLFE)

\begin{tabular}{ll}
\hline Begin & \\
Step 1 & Solve eq.(1)
\end{tabular}

Step 2 Solve eq. (2)

Step 2.1: $\quad$ Solve eq.(3)

Step 2.2: $\quad$ Solve eq.(4)

Step 2.3: $\quad$ Solve eq.(5) and concatenate eq (4)

\section{Step 3:}

Step 3.1: $\quad$ Compute eq. (6)

Step 3.2: $\quad$ Obtain facial similarity

End

Algorithm: Deep Learning Facial Features Learning

\section{Results and Discussions}

The results of the proposed method with respect to other contemporary efforts reported in different years is competitively good and has shown that the erected method based approach is most essential in applications that require to measure facial similarities.

\section{Comparison of Results}

The Deep Learning Facial features learning concatenation with Squeeze Net facial features learning phases have been assessed in the present work. The Concatenation of Processing with Squeeze Net facial features with Deep Neural Networks has made the system more efficient, the results of comparison of erected method with other contemporary methods mentioned in table 1 presents the comparison done by the proposed system with other methods.

Table 1. Comparison of Accuracies of contemporary methods with proposed approach

\begin{tabular}{cllll}
\hline \hline Year & Authors & Algorithms & Databases & Accuracy \\
\hline \hline \multirow{2}{*}{ Kohli et al. } & $\begin{array}{l}\text { Self-similarity } \\
\text { weber faces } \\
\text { Guo et a }\end{array}$ & $\begin{array}{l}\text { Prod of likelihood ratio on salient } \\
\text { features }\end{array}$ & Customized Database & 75.01
\end{tabular}


Zhou et al

2013

Dibeklioglu et al.

Lu.et.al

Yan.et.al

2014

Dehghan et al

Yan et.al

Liu.et.al

2015

2017

2018

2019

Alirezazadeh et al

Zhou.et.al

2016

Naman Kohli et.al

Qingyan Duan.et.al

Yong Yang.et.al

Miguel Bordallo Transfer Learning with Feature of Lopez.et.al Non-linear Mapping

A Tidjani.et.al

Diego Lelis.et.al

Youness

Mansar.et.al
Face Verific
Verification

Kinship Verification Based on linear Mapping

Pyramid of Gabor based gradient oriented features

Spatial features with temporal

Neighborhood repulsed learning with Multi-view

Metric of Discriminative Learning

Auto-encoders with discrimination

Prototype discriminative feature learning

Kinship verification with Inheritable Fisher Vector

Kinship Verification with Genetic Algorithm for feature selection

Similarity Learning with Ensemble Method

Representation learning for Kinship Verification (KVRL-fcDBN)

KinfaceW

KinfaceW

Uva-NEMO SMILE

Kinship Verification with Deep Learning Features

KinfaceW

Deep Learning for Kinship Verification

KinfaceW

79.48

Kinship prediction with Deep Neural Networks
KinfaceW

76.42
KinfaceW,

96.31

2019
Proposed Method
UB Kinface,

95.49
Cornell Kinface

The present research work has focused more towards identifying the facial similarities among relatives as well as the quantification of the similarity in terms of percentage values with various datasets such as KinfaceW, UB Kinface and Cornell Kinface. 


\section{Graphical Results}

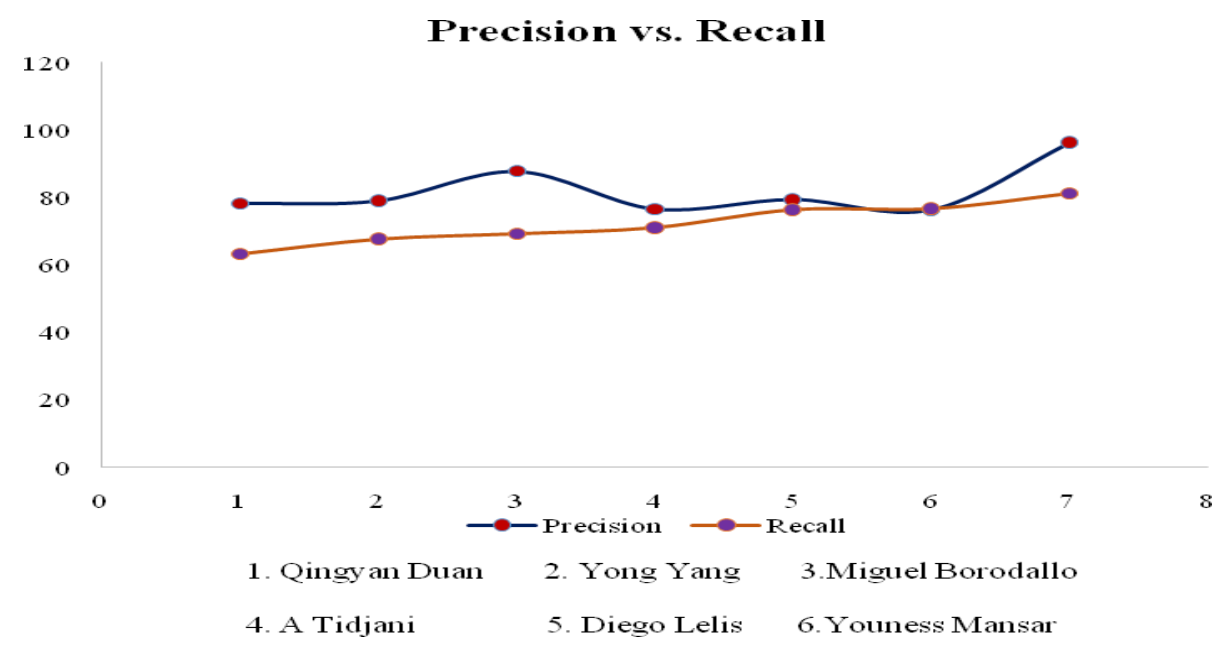

Figure 2. Comparison of precision vs. recall of different methods with respect to proposed Method

It is clear from the above fig. 2 that precision vs. recall of the erected method is comparatively better than other contemporary methods. The significance of the erected methods is very good to identify the facial similarities among different people presented in the dataset KinfaceW and UBKinface.

$$
\begin{aligned}
& \text { Precision }=\frac{T P}{T P+F P} \\
& \text { Recall }=\frac{T P}{T P+F N} \\
& \text { Accuracy }=\frac{T P+T N}{T P+T N+F P+F N}
\end{aligned}
$$

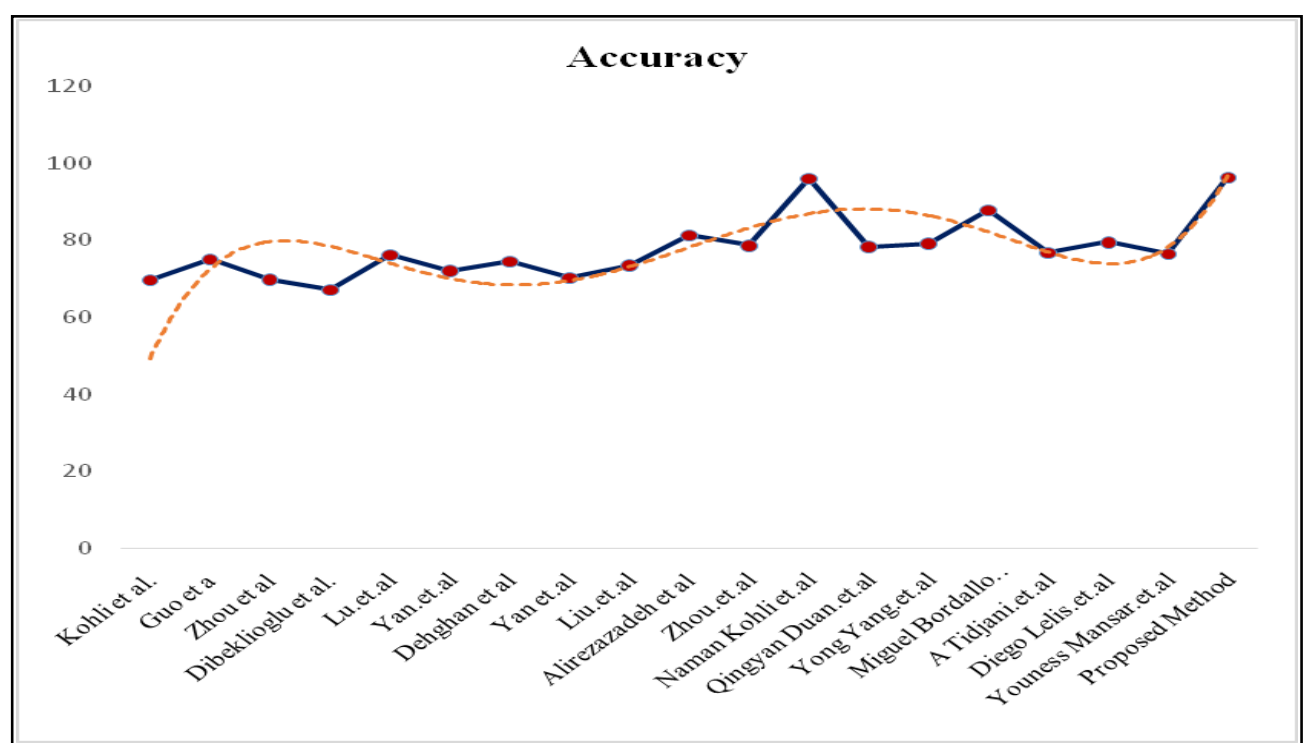

Figure 3. Comparison of accuracies of different methods with respect to proposed Method

The above fig. 3 presents the accuracy of the erected method with reference to other contemporary methods, The accuracy criteria is measured by analysing the True positivity obtained from the erected method with false positivity and false negativity of the proposed method determines the accurate classification of facial features 
into different classes of facial features represented in terms of performance metrics like Precision, Recall, Accuracy of the proposed method as per eq. (7), eq. (8), eq. (9) respectively.

\section{Conclusion}

The Deep Neural Network based facial features learning has contributed the performance of the proposed method by analysing and understanding the facial features of the dataset Kinface W and UB Kinface. The facial features of these dataset have been subjected to processing by deep neural network-based features learning and have yielded a good classification accuracy of $96.3 \%$ with respect to Father-Daughter, $96.1 \%$ with respect to Father-Son, $97.4 \%$ with respect to Mother-Daughter, and $96.5 \%$ with respect to Mother-Son relationships of images of KinfaceW-I and KinfaceW-II and UB Kinface datasets. The research work has focused its attention by contributing good classification accuracy with machine learning tasks for genealogical similarity.

\section{References}

1. Laiadi, Oualid \& Ouamane, Abdelmalik \& Boutellaa, Elhocine \& Benakcha, Abdel amid \& talebahmed, Abdelmalik \& Hadid, Abdenour. (2019). Kinship verification from face images in discriminative subspaces of color components. Multimedia Tools and Applications. 78. 10.1007/s11042-018-7027-9.

2. Xiuzhuang Zhou, Kai Jin, Min Xu, Guodong Guo, "Learning deep compact similarity metric for kinship verification from face images", Elsevier-Information Fusion, Vol. 48, 2019, pp.84-94.

3. Miguel Bordallo Lopez, Abdenour Hadid, Elhocine Boutellaa, Jorge Goncalves, Vassilis Kostakos, Simo Hosio, "Kinship Verification from Facial Images and Videos: Human vs. Machine”, pp. 873-890, Vol 29, Issue 5,2018.

4. Sanjay, H. S., Arundathi Hazare, and S. Bhargavi. "EEG based GAP perception in human beings." IJAER 10.2 (2015): 92-101

5. Haibin Yana, Junlin Hu "Video-based kinship verification using distance metric learning", ElsevierPattern Recognition, Vol.75, March 2018, pp.15-24.

6. Shahar Mahpod, Yosi Keller "Kinship verification using multiview hybrid distance learning”, ElsevierComputer Vision and Image Understanding, Vol.167, Feb-2018, pp.28-36.

7. Yan-Guo Zhao, Zhan Song, Feng Zheng, Ling Shao, "Learning a Multiple Kernel Similarity Metric for kinship verification”, Elsevier-Information Sciences, Vol.430-431,March 2018, pp.247-260.

8. N. Kohli, M. Vatsa, R. Singh, A. Noore and A. Majumdar, "Hierarchical Representation Learning for Kinship Verification," in IEEE Transactions on Image Processing, vol. 26, no. 1, pp. 289-302, Jan. 2017.

9. Mohammed Almuashi, Zaiton Mohd Hashim, Dzulkifli Mohamad, Mohammed Hazim, Alkawaz Aida Ali, "Automated kinship verification and identification through human facial images: a survey", January 2017, Volume 76, Issue 1, pp. 265-307.

10. S. Wang, J. P. Robinson and Y. Fu, "Kinship Verification on Families in the Wild with Marginalized Denoising Metric Learning," 2017 12th IEEE International Conference on Automatic Face \& Gesture Recognition (FG 2017), Washington, DC, 2017, pp. 216-221.

11. Haibin Yan, "Kinship verification using neighbourhood repulsed correlation metric learning" ElsevierImage and Vision Computing, Volume 60, April 2017, Pages 91-97.

12. Min $\mathrm{Xu}$ and Yuanyuan Shang, "Kinship Verification Using Facial Images by Robust Similarity Learning”, Mathematical Problems in Engineering, Vol. 2016, Article ID 4072323, 8 pages.

13. Xiuzhuang Zhou, Haibin Yan, Yuanyuan Shang, "Kinship verification from facial images by scalable similarity fusion", Elsevier-Neurocomputing, Vol. 197, 12 July 2016, Pages 136-142

14. X. Wu, E. Boutellaa, M. B. López, X. Feng and A. Hadid, "On the usefulness of color for kinship verification from face images," 2016 IEEE International Workshop on Information Forensics and Security (WIFS), Abu Dhabi, 2016, pp. 1-6.

15. H. Yan, J. Lu and X. Zhou, "Prototype-Based Discriminative Feature Learning for Kinship Verification," in IEEE Transactions on Cybernetics, vol. 45, no. 11, pp. 2535-2545, Nov. 2015.

16. J. Lu et al., "The FG 2015 Kinship Verification in the Wild Evaluation," 2015 11th IEEE International Conference and Workshops on Automatic Face and Gesture Recognition (FG), Ljubljana, 2015, pp. 17.

17. Alirezazadeh, A. Fathi and F. Abdali-Mohammadi, "A Genetic Algorithm-Based Feature Selection for Kinship Verification," in IEEE Signal Processing Letters, vol. 22, no. 12, pp. 2459-2463, Dec. 2015.

18. Lu Kou, Xiuzhuang Zhou, Min Xu, and Yuanyuan Shang, "Learning a Genetic Measure for Kinship Verification Using Facial Images", Mathematical Problems in Engineering, Vol. 2015, Article ID 472473, 5 pages. 
19. D. Li, H. Zhou and K. Lam, "High-Resolution Face Verification Using Pore-Scale Facial Features," in IEEE Transactions on Image Processing, vol. 24, no. 8, pp. 2317-2327, Aug. 2015.

20. Q. Liu, A. Puthenputhussery and C. Liu, "Inheritable Fisher vector feature for kinship verification," 2015 IEEE 7th International Conference on Biometrics Theory, Applications and Systems (BTAS), Arlington, VA, 2015, pp. 1-6.

21. J. Lu, X. Zhou, Y. Tan, Y. Shang and J. Zhou, "Neighbourhood Repulsed Metric Learning for Kinship Verification," in IEEE Transactions on Pattern Analysis and Machine Intelligence, vol. 36, no. 2, pp. 331-345, Feb. 2014.

22. H. Yan, J. Lu, W. Deng and X. Zhou, "Discriminative Multimetric Learning for Kinship Verification," in IEEE Transactions on Information Forensics and Security, vol. 9, no. 7, pp. 1169-1178, July 2014.

23. Wang and C. Kambhamettu, "Leveraging appearance and geometry for kinship verification," 2014 IEEE International Conference on Image Processing (ICIP), Paris, 2014, pp. 5017-5021.

24. J. Lu et al., "Kinship verification in the wild: The first kinship verification competition," IEEE International Joint Conference on Biometrics, Clearwater, FL, 2014, pp. 1-6.

25. Mohammad Ghahramani, Wei-Yun Yau, Eam Khwang Teoh, "Family verification based on similarity of individual family member's facial segments", May 2014, Volume 25, Issue 4, pp. 919-930.

26. Tiago F. Vieira, Andrea Bottino, Aldo Laurentini, Matteo De Simone, "Detecting siblings in image pairs", Springer-The Visual Computer, December 2014, Volume 30, Issue 12, pp. 1333-1345.

27. Hamdi Dibeklioglu, Albert Ali Salah, Theo Gevers; The IEEE International Conference on Computer Vision (ICCV), 2013, pp. 1497-1504

28. R. Fang, A. C. Gallagher, T. Chen and A. Loui, "Kinship classification by modelling facial feature heredity," 2013 IEEE International Conference on Image Processing, Melbourne, VIC, 2013, pp. 29832987.

29. Xiuzhuang Zhou and Junlin $\mathrm{Hu}$ and Jiwen Lu and Yuan Yuan Shang and Yong Guan, Kinship VF, "Kinship verification from facial images under uncontrolled conditions", ACM Multimedia, 2011

30. Ravi Kumar Y B, Narayanappa C K, "Triangular Similarities of Facial Features to Determine: The Relationships among Family Members", Journal of Advanced Computational Intelligence and Intelligent Informatics, Vol.22, No 3, 2018.

31. R. Fang, K. D. Tang, N. Snavely and T. Chen, "Towards computational models of kinship verification," 2010 IEEE International Conference on Image Processing, Hong Kong, 2010, pp. 15771580.

\section{Biographies of Authors}

Ravi Kumar Y B received M Tech from Visvesvaraya Technological University,
Belagavi in 2012 and B E in Computer Science \& Engineering in 2008. He is currently
working as Assistant Professor at department of Computer Science, Yuvarajas College,
University of Mysore, Mysore. His research interests include Image processing, Pattern
Classification, Machine Learning and Feature Representation Learning.

